\title{
Soft Tissue Paraspinal Inflammatory Malignant Fibrous Histiocytoma Presenting as a Lumbar Abscess
}

\author{
Emmanouil Liarmakopoulos ${ }^{\mathrm{a}}$ Pavlos Lampropoulos $^{\mathrm{a}}$ \\ Athanasios Marinis ${ }^{\mathrm{a}}$ Charalambos Markakis ${ }^{\mathrm{a}}$ \\ Kassiani Manoloudaki ${ }^{b}$ Andreas Chatzimarkou ${ }^{a}$ \\ Spyros Rizos ${ }^{\mathrm{a}}$
}

${ }^{a}$ First Department of Surgery, Departments of ${ }^{b}$ Pathology and 'Radiology,

'Tzaneion' General Hospital, Zanni and Afentouli STR, Piraeus, Greece

\section{Key Words}

Malignant fibrous histiocytoma, inflammatory type $\cdot$ Soft-tissue sarcoma

\begin{abstract}
The rarest subtype of malignant fibrous histiocytoma (MFH) is the inflammatory type, which due to its peculiar clinical presentation may mimic an infectious process such as an abscess. The rarity of this disease and the unique features of its presentation may mislead the surgeon with consequent catastrophic results for the patient. In this study, a case report of a 65 -year-old female patient with a soft-tissue inflammatory MFH presenting as a lumbar abscess and a review of the current literature are presented.
\end{abstract}

\section{Introduction}

Malignant fibrous histiocytoma (MFH) is the most common soft-tissue sarcoma of adults aged $>45$ years, involving most commonly the extremities and the retroperitoneum, with a slight male-gender predomination. The inflammatory type of MFH is the rarest of all and is characterized by a predominance of xanthoma cells with an intense neutrophilic infiltration. There is no consensus regarding the best diagnostic and treatment modality of inflammatory MFH, although most authors agree that surgery plays a pivotal role. We present a patient who was admitted to our hospital with a misdiagnosis of an abscess formation, although the patient suffered from a soft-tissue paraspinal MFH of the inflammatory type. 


\section{Case Presentation}

A 65-year-old female patient was admitted to our surgical department, complaining of a painful lumbar mass with high fever, which developed a few days after falling down the stairs. Physical examination revealed a temperature of $39^{\circ} \mathrm{C}$ and a palpable, protruding, fluctuating, and erythematous lumbar mass. Past medical history was unremarkable. Routine laboratory data on admission showed

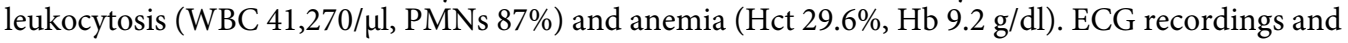
chest radiographs were normal.

Contrast-enhanced ultrasound (CEUS) revealed a hypoechoic mass of $9.8 \times 8 \times 11 \mathrm{~cm}$ with an irregular and heterogeneous structure and abundant vascularization. Having not excluded in our differential diagnosis an abscess formation, paracentesis was performed which revealed pus with abundant pyospheres and a negative culture. A wide drainage procedure was decided on, and sticky material was encountered along with extensive bleeding from the tumor site. Biopsy material was sent for pathological examination. Postoperatively, there was no clinical and biochemical improvement and our patient remained distressed, despite the use of wide spectrum intravenous antibiotics.

Abdominal CT scan was performed, and the report led to the diagnosis of a paraspinal abscess formation (fig. 1). Under ultrasound guidance, percutaneous drainage was attempted unsuccessfully due to the thick consistency of the tumor content. A repeat ultrasound examination showed pronounced vascularization inside an ill-defined structure with contrast material oozing from the paracentesis site, thus a sarcoma was suspected by the radiologists.

At that time, we obtained the biopsy results that showed malignant fibrous histiocytoma of inflammatory type. Immunohistochemistry revealed positive reactivity to CD68 and vimentin and negative reactivity to CKAE1, CKAE3, EMA, CD45, CD15, CD30, S100, HMB45, MELAN-A, actin, desmin, MVOD1, CD34, CD99, chromogranin, and synaptophysin (fig. 2). Our patient was scheduled for magnetic resonance imaging, which showed a large heterogeneous space-occupying lesion with lobular peripheral architecture at the anatomical site of the right sacrospinalis muscle, with signs of malignant infiltration of the right iliac bone (fig. 3 ).

After an oncological consultation meeting, the tumor was considered inoperable and the patient was scheduled for neoadjuvant radiotherapy. Unfortunately, excessive bleeding occurred that required multiple transfusions. Finally, our patient died 5 months after the initial presentation of the disease.

Written informed consent was obtained from the patient for publication of this case report and any accompanying images. A copy of the written consent is available for review by the Editor-in-Chief of this journal.

\section{Discussion}

$\mathrm{MFH}$ is the commonest type of soft-tissue sarcomas, representing $28 \%$ of all sarcomas. It was first described in 1964 as 'malignant fibrous xanthomas' after an extensive analysis of 53 cases which possessed histiocytic and fibroblastic elements with a storiform pattern, accompanied by inflammatory, xanthomatous, and pleomorphic giant cells [1]. MFHs occur more frequently in the extremities, followed by the abdominal cavity and the retroperitoneum [2], even though these tumors may be found in any part of the body, including the digestive tract, spermatic cord, kidney, pancreas, spleen, lung, thymus, ovaries, and gallbladder [2]. Inflammatory $\mathrm{MFH}$ is a rare subtype which was first described as 'inflammatory fibrous histiocytoma' [3], due to the intense acute inflammatory infiltrate in the absence of known infectious agents and unassociated with tissue necrosis. At present, inflammatory $\mathrm{MFH}$ is classified as a unique variant of $\mathrm{MFH}$ which commonly affects the retroperitoneum and, less frequently, the extremities [4].

Patients with retroperitoneal tumors develop constitutional symptoms including malaise, weight loss, anorexia, and abdominal discomfort. Apart from these symptoms, 
patients suffering from inflammatory MFH tumors present with fever, leukocytosis with neutrophilic or eosinophilic predomination and elevated serum C-reactive protein [5]. Thus, it is not unusual to consider an abscess formation in the differential diagnosis, as in our case. Some studies have documented that inflammatory MFHs have eosinophilic and neutrophilic chemotactic activity, which could potentially explain the blood chemistry profile and the abundant neutrophil infiltration of the tumor [6]. There are an increasing number of reports that implicate an association of accidental or surgical trauma and the pathogenesis of MFH [7]. This hypothesis applies to our case, since our patient had reported a recent fall at the site of the tumor. We believe that trauma plays an important role in the development of MFH, but further studies are needed for clarification.

Imaging modalities are helpful in providing data about the size, location, vasculature, extension of the tumor, and in designing preoperatively the extent of the surgical excision. Inflammatory MFH quite often present as well-defined lesions, with hypoechoic features, with or without intralesional calcifications at abdominal real-time ultrasound scanning [8]. Computer tomography scanning with intravenous contrast agents shows muscle density patterns, and, as occurred in our case, these tumors are mostly hypervascular with an early wash-out venous phase [9], even though less frequently, these tumors might be hypovascular due to tissue necrosis and hemorrhage [10]. Magnetic resonance imaging has not proved helpful in distinguishing between benign and malignant lesions. De Schepper et al. [11] found that the signs that had the greatest specificity and sensitivity for malignancy included 'absence of low signal intensity on T2', 'signal inhomogeneity on $\mathrm{T} 1$ ', and 'mean diameter of the lesion greater than $33 \mathrm{~mm}$ '. Kalayanarooj [12] concluded that features that favor malignancy with statistical significance were heterogeneous signal on T2-weighted, perilesional edema or invasion, and necrosis in the masses. Definitive diagnosis can be made only by biopsy, which should be taken at multiple sites to avoid misdiagnosis [4].

Surgical wide excision with tumor-free margins remains the principal modality with intent to cure. Given the fact that MFH has a poor prognosis with high local recurrence rates and distant metastasis especially with tumors larger than $10 \mathrm{~cm}$ [13], special attention has been given to the role of adjuvant therapies. Adjuvant therapy, until recently, has failed to improve survival, keeping the 5-year survival rate between 12 and $70 \%$ [14]. Chemotherapy is not effective and radiotherapy is limited by toxicity to the adjacent organs, thus aggressive successful en bloc resection of the primary tumor and local recurrences remains the most important prognostic factor [15].

\section{Conclusion}

Inflammatory MFH represents a unique subtype of MFH, which is extremely rare and quite often mimics an abscess formation due to the blood chemistry profile and its clinical presentation. It has a poor prognosis with high recurrence and low survival rates. Inflammatory MFH should be included in the differential diagnosis in patients with a large retroperitoneal mass and systemic inflammatory response syndrome, especially with a personal history of trauma at the tumor site. The best therapeutic approach is an aggressive wide surgical resection of the tumor mass en bloc. 


\section{Disclosure Statement}

The authors declare that they have no conflict of interests.

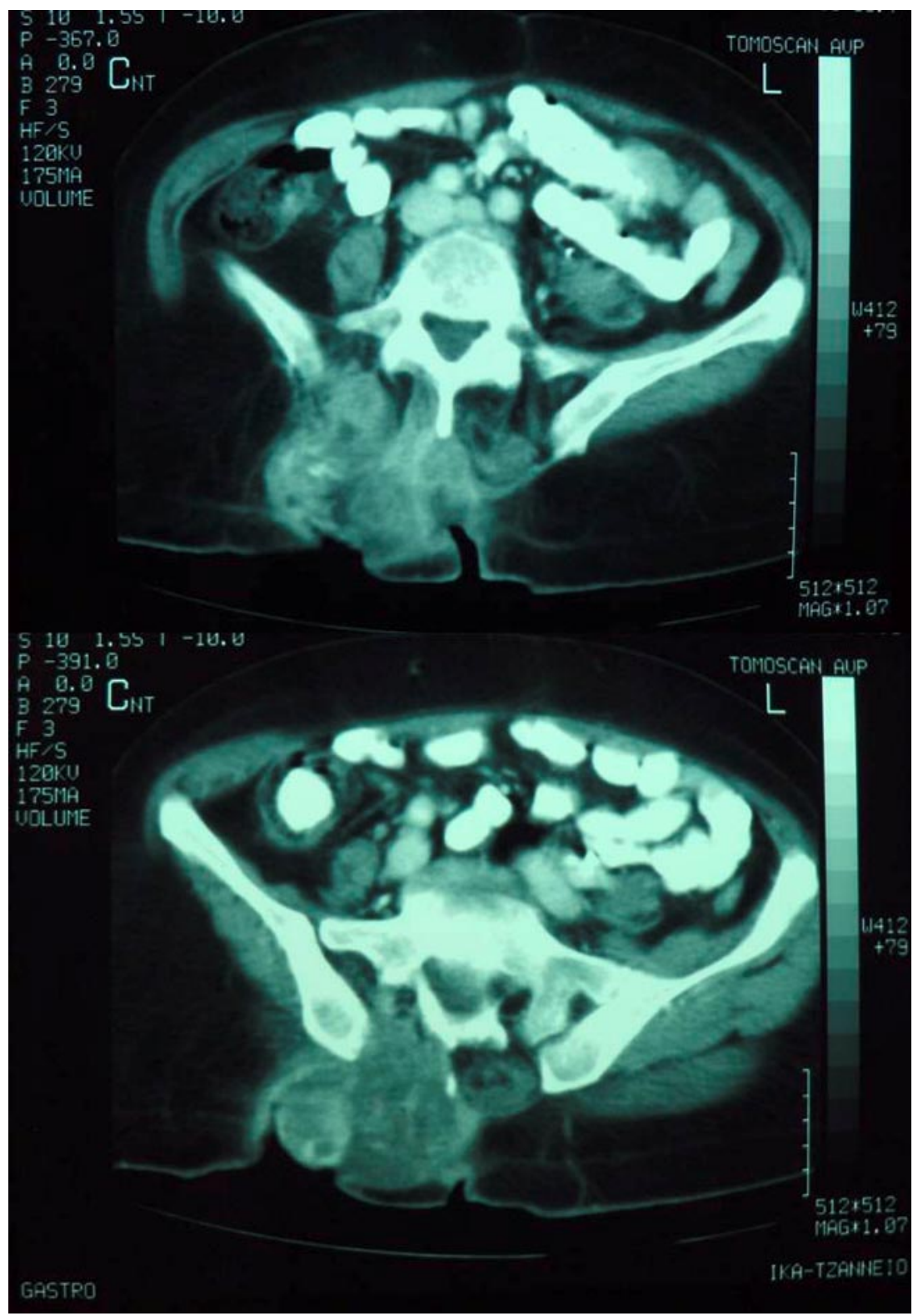

Fig. 1. An axial computed tomography demonstrating a right paraspinal abscess. 

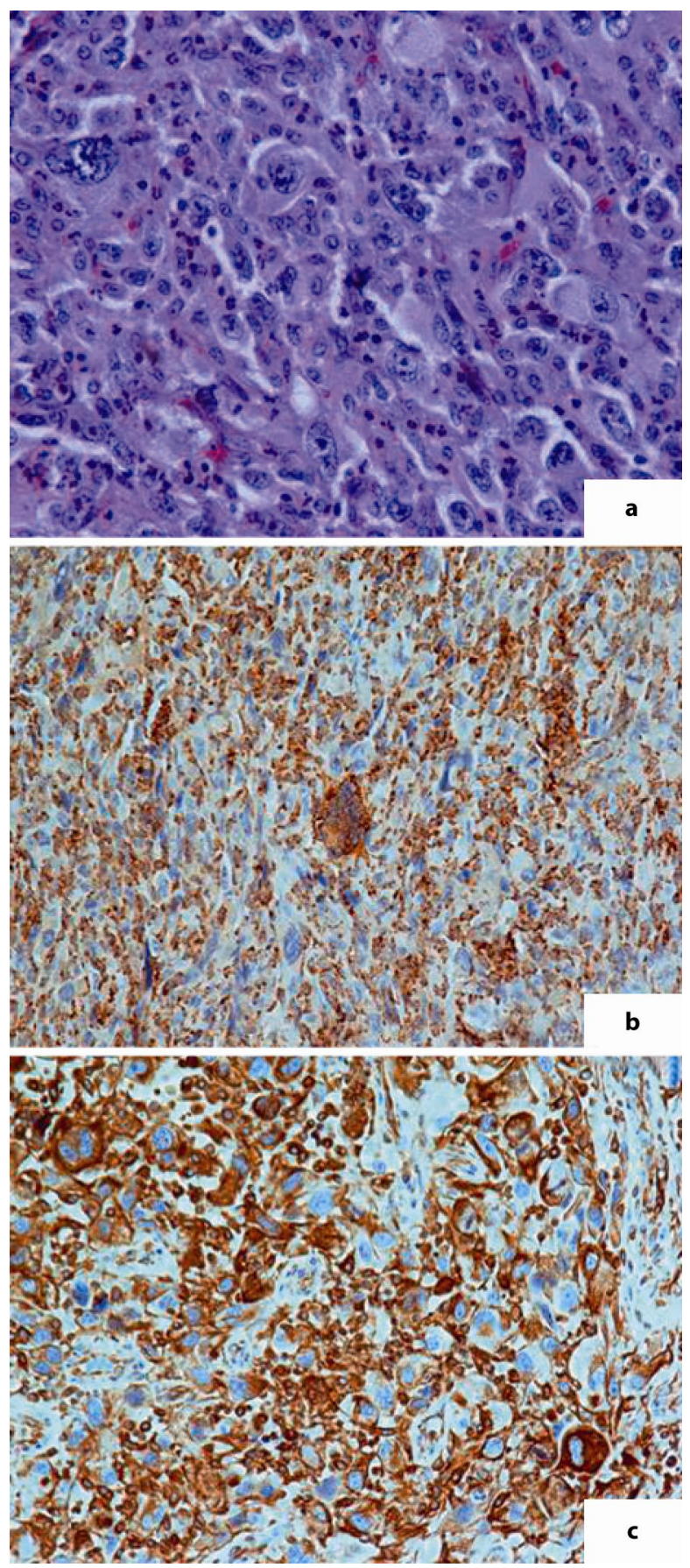

Fig. 2. a Phagocytic activity of neoplastic cells $(\mathrm{H} \& \mathrm{E}, \times 200)$; b positive reactivity of malignant xanthomatous cells $(\mathrm{CD} 68, \times 400)$; c positive reactivity of malignant cells (vimentin, $\times 200$ ). 


\begin{tabular}{c|l|l|l}
$\begin{array}{c}\text { Case Reports in } \\
\text { Oncology }\end{array}$ & $\begin{array}{l}\text { Case Rep Oncol 2011;4:343-349 } \\
\text { DOl: } 10.1159 / 000330367\end{array}$ & $\begin{array}{l}\text { Published online: } \\
\text { July 9, 2011 }\end{array}$ & $\begin{array}{l}\odot \text { 2011 S. Karger AG, Basel } \\
\text { ISSN 1662-6575 } \\
\text { www.karger.com/cro }\end{array}$ \\
\hline
\end{tabular}

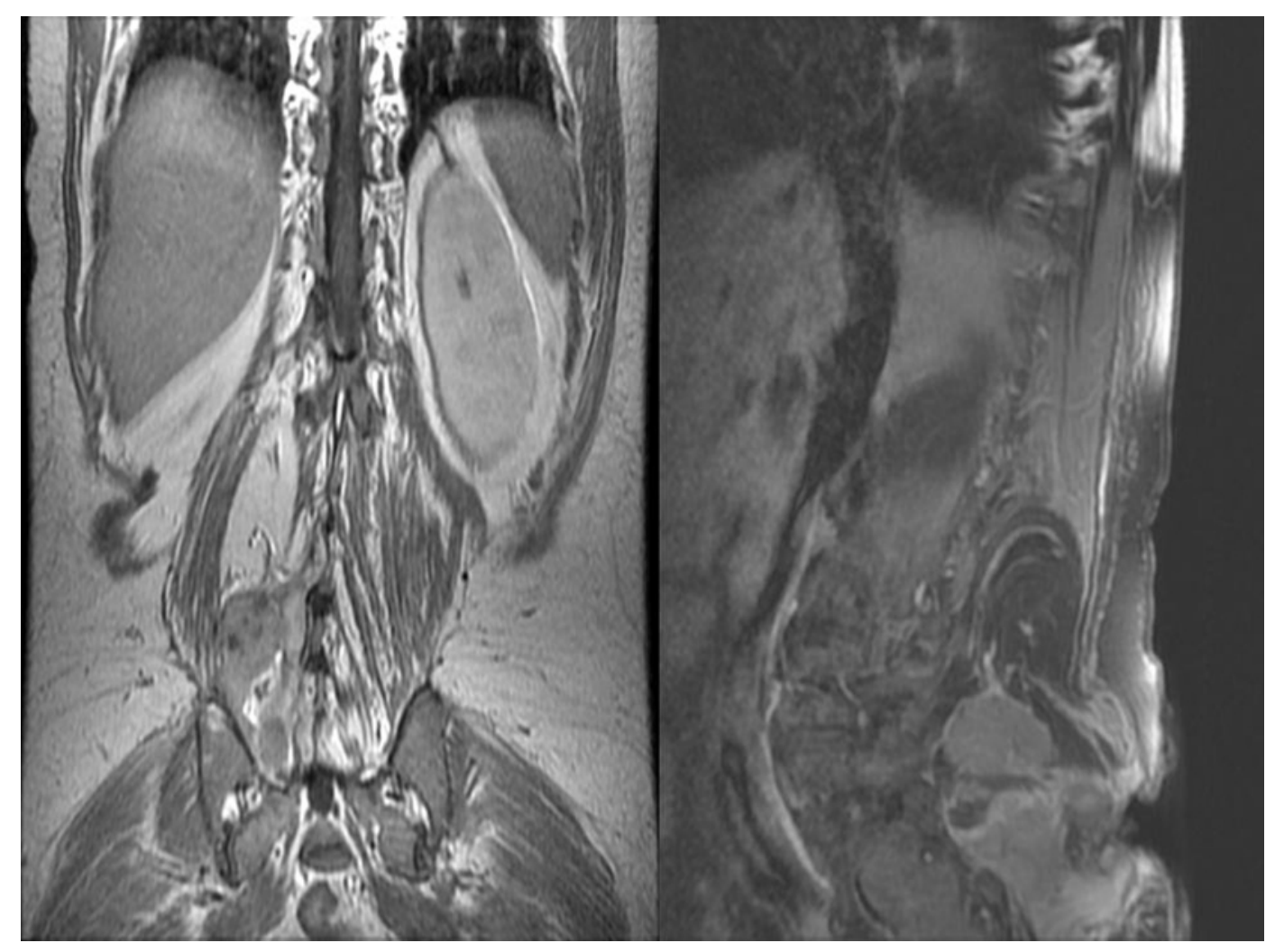

Fig. 3. Magnetic resonance imaging (sagittal and coronary views) demonstrating a large heterogeneous mass with lobular peripheral architecture at the anatomical site of the right sacrospinalis muscle, with malignant infiltration of the right iliac bone.

\section{References}

1 O'Brien JE, Stout AP: Malignant fibrous xanthomas. Cancer 1964;17:1445-1455.

-2 Weiss SW, Enzinger FM: Malignant fibrous histiocytoma. An analysis of 200 cases. Cancer 1978;41:2250-2266.

3 Kyriakos M, Kempson RL: Inflammatory fibrous histiocytoma, an aggressive and lethal lesion. Cancer 1976;37:1584-1606.

4 Enzinger FM, Wiess SW: Malignant fibrohistiocytic tumours; in Enzinger FM, Weiss SW (eds): Soft Tissue Tumors. Mosby, St. Louis, Washington, Toronto, 1988, pp 535-569.

5 Nakanishi H, Araki N, Kudawara I, Kuratsu S, Matsumine A, Mano M, Naka N, Myoui A, Ueda T, Yoshikawa $\mathrm{H}$ : Clinical implications of serum C-reactive protein levels in malignant fibrous histiocytoma. Int J Cancer 2002;99:167-170.

-6 Yoshida M, Matsuzaki H, Hata H, Matsuno F, Takeya M, Okabe H, Takatsuki K: Neutrophil chemotactic factors produced by malignant fibrous histiocytoma cell lines. Br J Cancer 1993;67:508-513.

7 Joss R, Ganz R, Ryssel HJ, Remagen W, Brunner K: Posttraumatic soft tissue sarcoma: a case study of a malignant fibrous histiocytoma of the elbow joint which appeared six and a half years after a severe injury. Schweiz Med Wochenschr 1980;110:2021-2024.

-8 (No authors listed:) Hypervascular retroperitoneal mass in a patient with Fever and leukocytosis - contrastenhanced ultrasonographic findings in a case of inflammatory malignant fibrous histiocytoma (in German). Ultraschall Med 2011;32:1-4.

-9 Ros PR, Viamonte M Jr, Rywlin AM: Malignant fibrous histiocytoma: mesenchymal tumor of ubiquitous origin. AJR Am J Roentgenol 1984;142:753-759. 
10 Burgener FA, Landman S: Angiographic features of malignant fibrous histiocytomas. Radiology 1976;121:581583.

11 De Schepper AM, Ramon FA, Degryse H: Statistical analysis of MRI parameters predicting malignancy in 141 soft tissue masses. Rofo 1992;156:587-591.

12 Kalayanarooj S: Benign and malignant soft tissue mass. Magnetic resonance imaging criteria for discrimination. J Med Assoc Thai 2008;91:74-81.

13 Pezzi CM, Rawlings MS Jr, Esgro JJ, Pollock RE, Romsdahl MM: Prognostic factors in 227 patients with malignant fibrous histiocytoma. Cancer 1992;69:2098-2103.

$\checkmark 14$ Pirayesh A, Chee Y, Helliwell TR, Hershman MJ, Leinster SJ, Fordham MV, Poston GJ: The management of retroperitoneal soft tissue sarcoma: a single institution experience with a review of the literature. Eur J Surg Oncol 2001;27:491-497.

15 Lewis JJ, Leung D, Woodruff JM, Brennan MF: Retroperitoneal soft-tissue sarcoma: analysis of 500 patients treated and followed at a single institution. Ann Surg 1998;228:355-365. 